\title{
(Sub)millimetre emission from NGC 1569: An abundance of very small grains
}

\author{
U. Lisenfeld ${ }^{1}$, F. P. Israel ${ }^{2}$, J. M. Stil ${ }^{2,3}$, and A. Sievers ${ }^{1}$ \\ 1 IRAM, Avendida Divina Pastora 7, N.C., 18012 Granada, Spain \\ 2 Sterrewacht Leiden, Postbus 9513, 2300 RA Leiden, The Netherlands \\ 3 Physics Department, Queen's University, Kingston ON K7L 4P1, Canada
}

Received 18 May 2001 / Accepted 5 December 2001

\begin{abstract}
We present new data of the dwarf galaxy NGC 1569 at $450 \mu \mathrm{m}, 850 \mu \mathrm{m}$ and $1200 \mu \mathrm{m}$ taken with SCUBA at the JCMT and the bolometer array at the IRAM $30 \mathrm{~m}$ telescope. After including data from IRAS at 12, 25, 60 and $100 \mu \mathrm{m}$, we have successfully fitted the dust grain population model of Désert et al. (1990) to the observed midinfrared-to-millimetre spectrum. The fit requires a combination of both large and very small grains exposed to a strong radiation field as well as an enhancement of the number of very small grains relative to the number of large grains. We interpret this as the consequence of large grain destruction due to shocks in the turbulent interstellar medium of NGC 1569. The contribution of polyaromatic hydrocarbons (PAH's) is found to be negligible. Comparison of the dust emission maps with an HI map of similar resolution shows that both dust and molecular gas distributions peak close to the radio continuum maximum and at a minimum in the HI distribution. From a comparison of these three maps and assuming that the gas-to-dust mass ratio is the same everywhere, we estimate the ratio of molecular hydrogen column density to integrated CO intensity to be about 25-30 times the local Galactic value. The gas-to-dust ratio is 1500-2900, about an order of magnitude higher than in the Solar Neighbourhood.
\end{abstract}

Key words. galaxies: individual: NGC 1569 - galaxies: ISM - galaxies: irregular - ISM: dust, extinction

\section{Introduction}

Dwarf galaxies characteristically have low metallicities and consequently low dust abundances. In dwarf galaxies, both dust properties and amounts differ from those in spiral galaxies, as for instance implied by a difference in IRAS colours (cf. Melisse \& Israel 1994). However, actual dust abundances and dust composition are only poorly known for dwarf galaxies. The dust mass of a galaxy can be estimated reliably only if good (sub)mm measurements allow to constrain the amounts of relatively cold dust which may dominate the total dust mass with only a very limited contributions to the emission at infrared wavelengths. Such data are available for a limited number of galaxies, and are especially scarce for faint dwarf galaxies.

NGC 1569 (Arp 210; VII Zw 16) is a nearby irregular dwarf galaxy at a distance of $2.2 \mathrm{Mpc}$ (Israel 1988, hereafter I88). It is a member of the low-galactic latitude IC 342/Maffei 1/Maffei 2/Dw 1 group, containing at least 15 dwarf galaxies (Huchtmeier et al. 2000). NGC 1569 is presently in the aftermath of a massive burst of star formation (I88; Israel \& De Bruyn 1988; Waller 1991). Its

Send offprint requests to: U. Lisenfeld, e-mail: ute@iram.es present star formation rate, derived from the $\mathrm{H} \alpha$ luminosity, is $0.4 M_{\odot} \mathrm{yr}^{-1}$ (Waller 1991). In the recent past, this galaxy has experienced a starburst which started about $1-2 \times 10^{7}$ yr ago as estimated by Israel (I88) and about $1.5 \times 10^{7}$ yr ago determined by Vallenari \& Bomans (1996) from colour-magnitude diagrams. The end of the starburst, about 5 Myr ago, can be well dated by a kink in the synchrotron spectrum (Israel \& De Bruyn 1988) and photometric studies (Vallenari \& Bomans 1996; Greggio et al. 1998). Although a small galaxy with neutral atomic hydrogen (HI) dimensions of $3 \times 2 \mathrm{kpc}$ (Israel \& Van Driel 1990; Stil \& Israel, in preparation, hereafter SI01), NGC 1569 contains two extremely compact luminous star clusters A and B (Ables 1971; Arp \& Sandage 1985; Aloisi et al. 2001 and references therein) with bolometric luminosities of order $10^{8} L_{\odot}$ located in a deep HI minimum. A third such cluster is embedded in bright emission nebulosity (F.P. Israel \& W. Wamsteker, unpublished; Prada et al. 1994; listed as No. 10 by Hunter et al. 2000) coincident with the peak of the radio continuum distribution.

The HI observations show the galaxy to be in solidbody rotation out to a radius of $1^{\prime}(0.64 \mathrm{kpc})$; however, much of the HI is in chaotic motion (SI01). To the east of 
NGC 1569, an apparently counterrotating HI cloud connected by an HI bridge to NGC 1569 is observed (Stil \& Israel 1998). The brightest HI with column densities $N(\mathrm{HI}) \approx 2 \times 10^{20} \mathrm{~cm}^{-2}$, occurs in the three main peaks of an $\mathrm{HI}$ ridge in the northern half of the galaxy. About a third of the total mass of NGC 1569 resides in its neutral atomic hydrogen (I88, SI01). Weak CO emission is found close to the radio continuum, between two HI maxima. It was mapped in the $J=1-0$ and $J=2-1$ transitions by Greve et al. (1996). Comparison with the $J=1-0$ CO detection in a larger beam by Taylor et al. (1998) suggests that the maps by Greve et al. contain virtually all CO emission from the western half of NGC 1569 . Aperture synthesis maps of the same two CO transitions with the IRAM interferometer show distinct $\mathrm{CO}$ clouds of sizes ranging from 40 to $100 \mathrm{pc}$ (Taylor et al. 1999). As these maps recover only a quarter of the single-dish flux, weak and more diffusely distributed $\mathrm{CO}$ must be present. $\mathrm{CO}(3-2)$ observations show a high $J=3-2 / J=2-1$ ratio of 1.4 (Mühle et al. 2001) indicating a warm molecular gas phase.

The far-infrared emission from NGC 1569, observed with IRAS, is remarkably strong for a dwarf galaxy; the continuum spectrum indicates that the dust in the galaxy is exposed to intense radiation fields (I88).

In this paper we extend the far-infrared spectrum of NGC 1569 to (sub)millimeter wavelengths by presenting new observations obtained with the IRAM and JCMT telescopes. These new observations allow us, for the first time, to determine the amount of dust in this galaxy and to study its properties in detail.

\section{Observations}

Observations at $850 \mu \mathrm{m}$ and at $450 \mu \mathrm{m}$ were made in October 1997 and again in March 2000 with the SCUBA camera at the JCMT on Mauna Kea (Hawaii). As the quality of the later observations greatly surpasses that of the earlier, we only discuss the March 2000 observations in this paper. SCUBA consists of two bolometer arrays of 91 elements at $450 \mu \mathrm{m}$ and 37 elements at $850 \mu \mathrm{m}$, both with a field of view of about 2.3' (Holland et al. 1999), thus covering the whole galaxy. Both wavelengths were observed simultaneously in jiggle mode. The total integration times, half on source, half on sky, was 5 hrs. The images were chopped with a throw of $120^{\prime \prime}$ at a frequency of $7.8 \mathrm{~Hz}$.

We applied the standard reduction procedure: this includes flat-fielding, removal of transient spikes, correction of atmospheric opacity $\left(\tau_{850} \approx 0.3 ; \tau_{450} \approx 1.1\right)$; pointing correction and sky-removal. The data were calibrated by observation of the standard source CRL 618 $\left(F_{850}=11.2 \mathrm{Jy}, F_{450}=4.56 \mathrm{Jy}\right)$ immediately before and after the NGC 1569 observations. From the calibrator images, we determined a beam-size (FWHM) of $15.3 \times 15.6^{\prime \prime}$ at $850 \mu \mathrm{m}$.

Observations at $1200 \mu \mathrm{m}$ were made in December 1998 at the IRAM $30 \mathrm{~m}$ telescope on Pico Veleta (Spain), with the 19-channel bolometer array of the Max-PlanckInstitut für Radioastronomie (MPIfR). Additional maps at $1200 \mu \mathrm{m}$ were obtained in April 1999 and again in March 2000 with the 37-channel bolometer of the MPIfR. The beam-size is $10.8^{\prime \prime}$. The observations were done onthe-fly, with a wobbler throw of $46^{\prime \prime}$. Opacities ranged from 0.1 to 0.3 . The data were reduced in a standard manner including baseline subtraction, spike removal and sky noise removal. The maps were calibrated by observation of the planets Mars and Uranus.

\section{Integrated flux-densities}

Maps of NGC 1569 at the three observed wavelengths are shown in Figs. 1 and 2. We have optimized the SCUBA dataset by excluding the two samples out of ten that were taken under poor atmospheric opacity conditions. The noise levels of the maps are $2.5 \mathrm{mJy} /$ beam $(1200 \mu \mathrm{m})$, $4 \mathrm{mJy} /$ beam $(850 \mu \mathrm{m})$ and $68 \mathrm{mJy} /$ beam $(450 \mu \mathrm{m})$.

We determined total galaxy flux-densities by integrating the maps over increasingly larger areas until the cumulative flux-densities thus obtained converged to a final value. However, it was found that low-level emission from NGC 1569 extended over most of the SCUBA field of view rendering an accurate determination of the (sky) zerolevel questionable. This was not a problem in the larger IRAM $1200 \mu \mathrm{m}$ field. For this reason, we fitted the $850 \mu \mathrm{m}$ and $450 \mu \mathrm{m}$ cumulative growthcurves to the scaled growthcurve at $1200 \mu \mathrm{m}$, allowing for a zerolevel offset of the $850 \mu \mathrm{m}$ and $450 \mu \mathrm{m}$ growthcurves. The scaling of the $1200 \mu \mathrm{m}$ growthcurve was uniquely defined by the requirement that the difference between the growthcurves increases as the square of the radius, as expected if the SCUBA maps had a zerolevel offset.

In this way, we obtained total flux-densities $S_{1200}=$ $250 \pm 30 \mathrm{mJy}, S_{850}=410 \pm 45 \mathrm{mJy}$ and $S_{450}=1820 \pm$ $700 \mathrm{mJy}$ (Table 1). The errors quoted are formal errors based on map noise and including, at $450 \mu \mathrm{m}$ and $850 \mu \mathrm{m}$, the error introduced by the zerolevel correction. The latter error is, however, small: from the goodness of the fit we estimate it to be about $4 \%$ at both $450 \mu \mathrm{m}$ and $850 \mu \mathrm{m}$. The actual uncertainty is larger because of calibration uncertainties. In particular at $450 \mu \mathrm{m}$, the telescope error pattern and relatively high atmospheric opacities conspire to produce a large uncertainty at this wavelength. We estimate the total error, including opacity correction, calibration, noise of the map and zerolevel correction to be about $50 \%$ at $450 \mu \mathrm{m}$ and $30 \%$ at both $850 \mu \mathrm{m}$ and $1200 \mu \mathrm{m}$.

Especially at the longer wavelengths of $850 \mu \mathrm{m}$ and $1200 \mu \mathrm{m}$, the broad-band flux-densities determined from the maps may contain non-negligible contributions by thermal free-free continuum emission and $\mathrm{CO}$ line emission. The contribution of the CO line to $1200 \mu \mathrm{m}$ broadband emission can be estimated from the $J=2-1$ CO measurements by Greve et al. (1996). Adding up all the emission detected by them, we obtain $I_{\mathrm{CO}}=$ $13.6 \mathrm{~K} \mathrm{~km} \mathrm{~s}^{-1}$ at $230 \mathrm{GHz}$. Allowing for the presence of a similar amount of undetected $\mathrm{CO}$ emission in the 


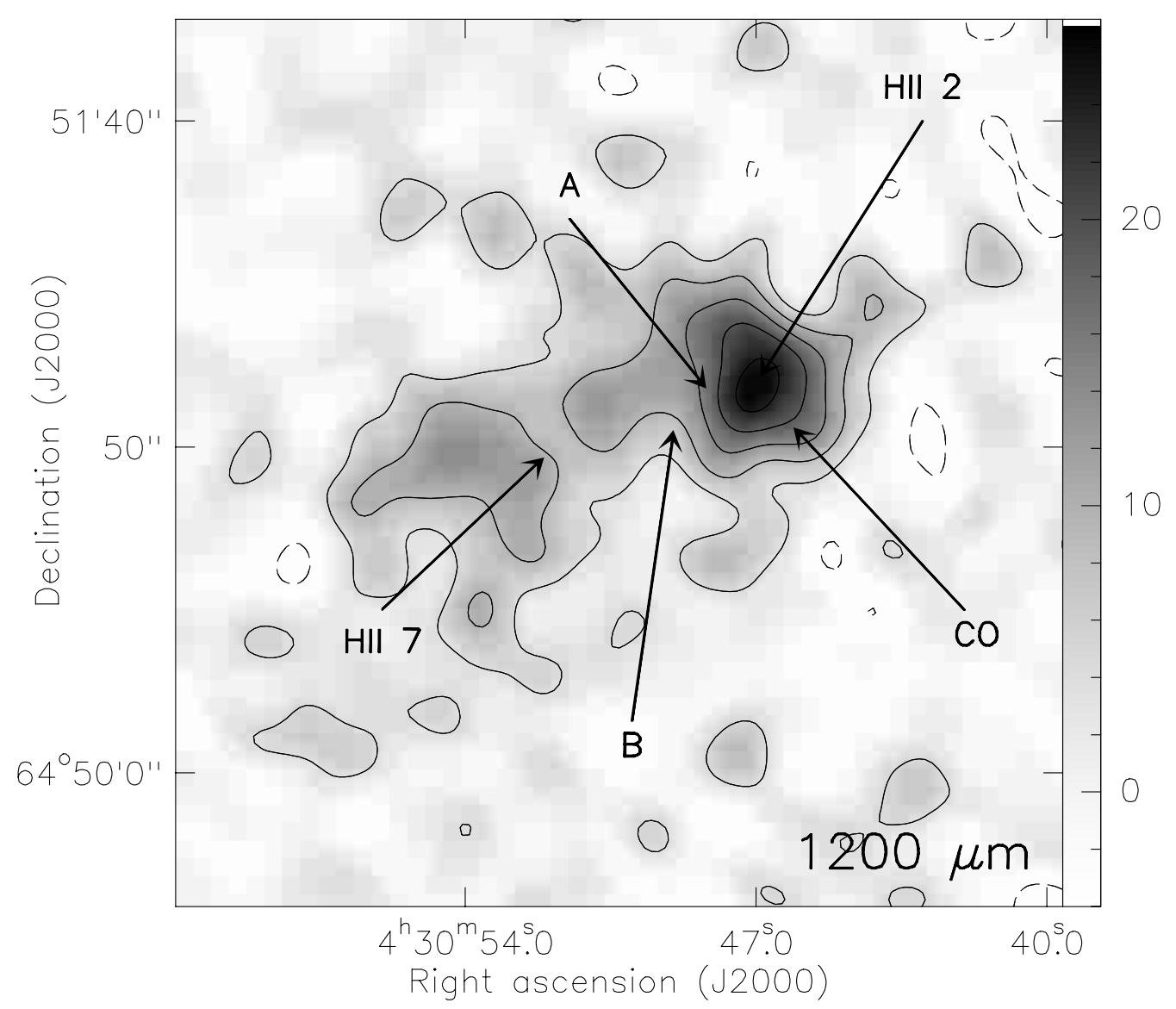

Fig. 1. Map of NGC 1569 at $1200 \mu \mathrm{m}$, smoothed to a resolution of $13^{\prime \prime}$. Contour values are at levels $-n, n, 2 n, 3 n \ldots .$, where $n=5 \mathrm{mJy} /$ beam. The lowest contour corresponds to $2 \sigma$, where $\sigma$ is the noise level of the map. The positions of the starclusters $\mathrm{A}$ and $\mathrm{B}$ are indicated, as well as the maximum of the CO distribution (Tayler et al. 1998) and the position of the two most prominent HII regions, catalogued as number 2 and 7 by Waller (1991).

remainder of NGC 1569 and using a conversion factor between $T_{\mathrm{mb}}$ and flux density of $4.6 \mathrm{Jy} / \mathrm{K}$ and an adopted instrumental bandwidth of $50 \mathrm{GHz}$ for the bolometer, we obtain a maximum of $2.1 \mathrm{mJy}$ contributed by the $J=2-1$ $\mathrm{CO}$ line to the total emission in the $1200 \mu \mathrm{m}$ band. The $J=3-2$ CO emission, contributing to the $850 \mu \mathrm{m}$ broadband emission, has been measured by Meier et al. (2001). At the CO peak in the map by Greve et al. (1996), they find the $J=3-2 / J=1-0$ ratio to be unity, characteristic of rather hot and dense molecular gas. From this result, we estimate a maximum contribution of $10 \mathrm{mJy}$ to the emission in the $70 \mathrm{GHz}$ wide SCUBA band at $850 \mu \mathrm{m}$. We thus find that, at either wavelength, contributions by $\mathrm{CO}$ to the total emission of NGC 1569 are unimportant, reflecting the general weakness of $\mathrm{CO}$ emission from dwarf galaxies.

Consideration of the thermal free-free continuum contribution does not warrant a similar conclusion. Its intensity has been estimated from radio maps at 1.5, 5, 8.4 and $15 \mathrm{GHz}$ by Wilding (1990). The derived total thermal radio continuum flux-density at $1 \mathrm{GHz}$ is $100 \mathrm{mJy}$, in very good agreement with the value of $97 \mathrm{mJy}$ estimated by Israel \& De Bruyn (1988) from reddening-corrected $\mathrm{H} \alpha$ measurements. Scaling this emission to higher frequencies (shorter wavelengths) as $\nu^{-0.1}$, we derive considerable thermal free-free contributions to the total emission of 58 , 56 and $52 \mathrm{mJy}$ at 1200,850 and $450 \mu \mathrm{m}(23 \%, 13 \%$ and $3 \%)$ respectively. In Table 1, we have summarized these results, together with further photometric data from the literature.

\section{Nature of the dust emission}

\subsection{Observed emission spectrum}

The dust emission and extinction in our Galaxy can be modelled as the combined effect of three components (e.g. Désert et al. 1990 - hereafter DBP90; Siebenmorgen \& Krügel 1992). These components are: (i) large grains obeying a power-law size distribution (cf. Mathis et al. 1977), (ii) polyaromatic hydrocarbons (PAH's) responsible for mid-infrared continuum and spectral feature emission, (iii) very small grains (VSG's) with sizes of a few $\mathrm{nm}$. The large grains are in equilibrium with the radiation field and their emission $F_{\lambda}$ can be described by a modified black-body curve following from the Kirchhoff-law: $F_{\lambda}=B(\lambda, T) k_{\lambda}$, where $B(\lambda, T)$ is the Planck function, $\lambda$ 

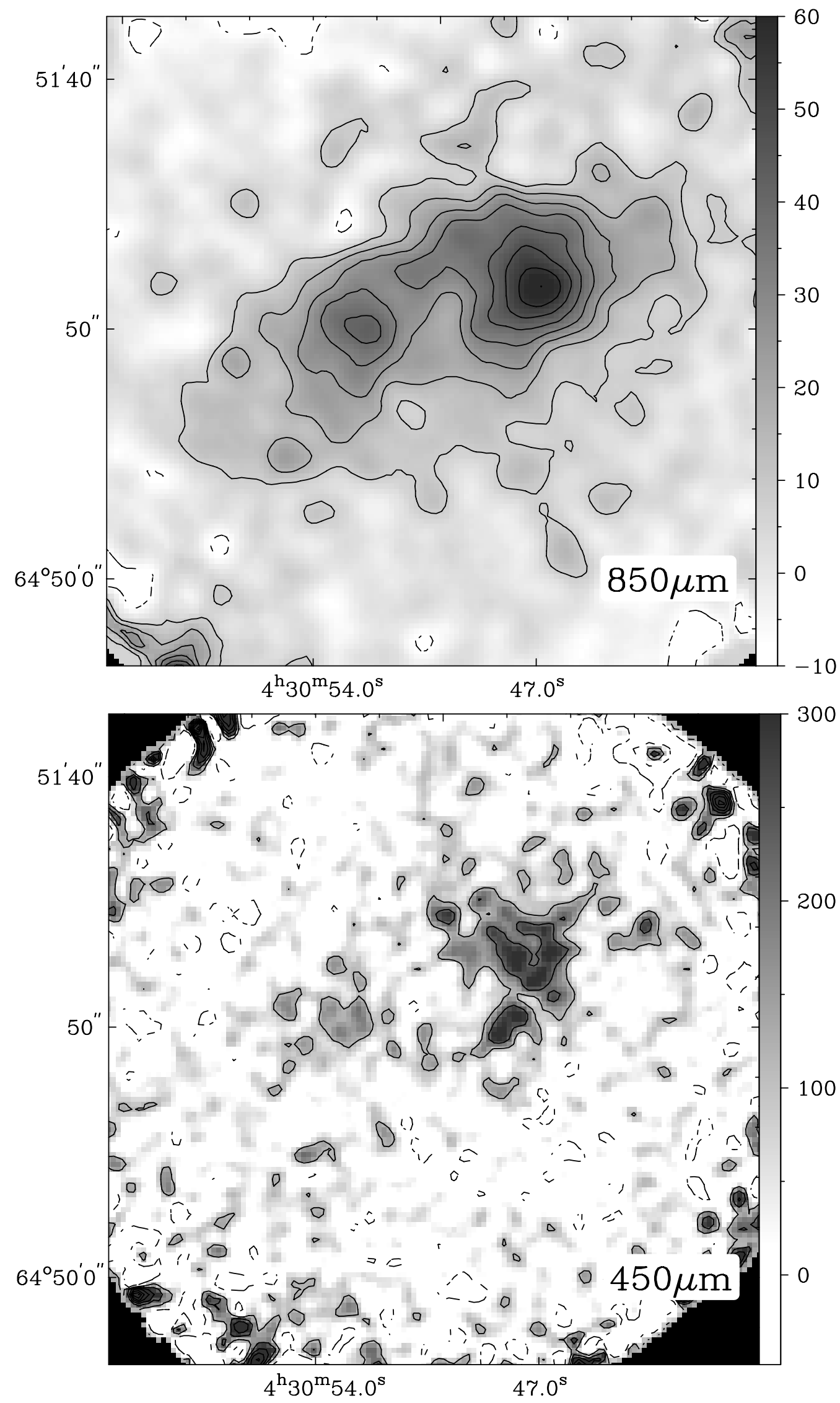

Fig. 2. Maps of NGC 1569 at 850 and $450 \mu \mathrm{m}$. Contour values are at levels $-n, n, 2 n, 3 n \ldots$. , where $n=8 \mathrm{mJy} / \mathrm{beam}(850 \mu \mathrm{m})$ and $n=115 \mathrm{mJy} /$ beam $(450 \mu \mathrm{m})$. The lowest contours correspond to $2 \sigma$, where $\sigma$ is the noise level of the map. 
Table 1. NGC 1569 emission from UV to millimetre.

\begin{tabular}{rcc}
\hline \multicolumn{3}{c}{ UV and optical flux density } \\
$\begin{array}{c}\text { flux density } \\
(\AA)\end{array}$ & $10^{-14} \mathrm{erg} \mathrm{s}^{-1} \mathrm{~cm}^{-1} \AA^{-1}$ & Ref. \\
\hline 1500 & $152 \pm 48$ & $(1)$ \\
1800 & $208 \pm 25$ & $(1)$ \\
2200 & $146 \pm 25$ & $(1)$ \\
2500 & $100 \pm 18$ & $(1)$ \\
3650 & $108 \pm 10$ & $(2)$ \\
4400 & $101 \pm 9$ & $(2)$ \\
5500 & $70 \pm 6$ & $(2)$
\end{tabular}

\begin{tabular}{rrrrr}
\hline \multicolumn{5}{c}{ Integrated flux density from $12 \mu \mathrm{m}$ to $1200 \mu \mathrm{m}$} \\
$\lambda$ & $\begin{array}{rrrr}\text { Observed } \\
(\mu \mathrm{m})\end{array}$ & $\begin{array}{r}\text { Thermal+CO } \\
(\mathrm{Jy})\end{array}$ & $\begin{array}{r}\text { Dust } \\
(\mathrm{Jy})\end{array}$ & Ref. \\
\hline 12 & 0.59 & - & $0.59 \pm 0.06$ & $(3)$ \\
25 & 5.95 & - & $5.95 \pm 0.6$ & $(3)$ \\
60 & 44.6 & - & $44.6 \pm 4.5$ & $(3)$ \\
100 & 52.2 & - & $52.2 \pm 5.2$ & $(3)$ \\
155 & 36 & - & $36 \pm 11$ & $(4)$ \\
450 & 1.82 & 0.052 & $1.77 \pm 0.88$ & $(5)$ \\
850 & 0.41 & 0.066 & $0.34 \pm 0.10$ & $(5)$ \\
1200 & 0.25 & 0.060 & $0.19 \pm 0.06$ & $(5)$ \\
\hline
\end{tabular}

(1) I88, corrected for Galactic foreground extinction as described therein.

(2) De Vaucouleurs et al. (1991), corrected for Galactic foreground extinction based on $E(B-V)$ (I88) and a standard solar neighbourhood reddening law as in I88.

(3) IRAS point source catalogue, flux densities are colourcorrected as described therein.

(4) Hunter et al. (1989).

(5) This work.

the wavelength, $T$ the dust temperature and $k_{\lambda}$ the extinction coefficient. In contrast, the VSG's are not at all in equilibrium. Their heat capacity is so limited that they can be heated to very high temperatures $(T \approx 1000 \mathrm{~K})$ by the absorption of a single photon. However, as they cool down rapidly, an ensemble of small grains cannot be described by a single temperature but rather by a relatively broad temperature distribution. Consequently, its integrated emission spectrum is significantly broader than that of large grains with a limited temperature range. Because the small-grain temperature is on average higher, this spectrum peaks in the mid-infrared. The wavelength dependence of $k_{\lambda}$ is not well known, however, models of interstellar dust that are both astronomically realistic in composition and able to reproduce the observed dust extinction and emission curves, predict $\beta \simeq 2$ for large grains (e.g. Draine \& Lee 1984; Ossenkopf \& Henning 1994; Krügel \& Siebenmorgen 1994). Observations of actively star-forming galaxies, for which the dust emission beyond about $60 \mu \mathrm{m}$ can be well described by only one temperature component, confirm a value of $\beta$ close to 2 (e.g. Chini et al. 1992; Krügel et al. 1998). A more general result has been obtained by Dunne et al. (2001) who have derived on a statistical basis $\beta \simeq 2$ for a sample of nearby galaxies with 450 and $850 \mu \mathrm{m}$ data. For small amorphous
Table 2. Flux densities of the various dust components.

\begin{tabular}{|c|c|c|c|c|c|}
\hline \multicolumn{4}{|c|}{ Model A: Cold dust } & \multicolumn{2}{|c|}{ Model B: Very small grains } \\
\hline $\begin{array}{l}\lambda \\
(\mu \mathrm{m})\end{array}$ & $\begin{array}{l}S_{\text {hot }} \\
(\mathrm{Jy})\end{array}$ & $\begin{array}{l}S_{\text {warm }} \\
(\mathrm{Jy})\end{array}$ & $\begin{array}{l}S_{\text {cold }} \\
\text { (Jy) }\end{array}$ & $\begin{array}{l}S_{\mathrm{VSG}} \\
(\mathrm{Jy})\end{array}$ & $\begin{array}{l}S_{\text {large grains }} \\
(\mathrm{Jy})\end{array}$ \\
\hline 12 & 0.68 & - & - & 0.44 & - \\
\hline 25 & 5.7 & 0.18 & - & 6.07 & 0.16 \\
\hline 60 & 1.9 & 41.2 & - & 26.2 & 18.1 \\
\hline 100 & 0.46 & 52.5 & - & 25.3 & 26.6 \\
\hline 155 & 0.11 & 26.6 & 0.02 & 17.2 & 14.1 \\
\hline 450 & - & 1.1 & 0.70 & 1.9 & 0.54 \\
\hline 850 & - & 0.12 & 0.26 & 0.39 & 0.05 \\
\hline 1200 & - & 0.03 & 0.11 & 0.16 & 0.01 \\
\hline
\end{tabular}

grains, a lower value of $\beta \simeq 1$ can perhaps be expected (Seki \& Yamamoto 1989; Tielens \& Allamandola 1987).

The integrated spectrum from $12 \mu \mathrm{m}$ to $1200 \mu \mathrm{m}$ of NGC 1569 provides important clues for the origin of the emission and for the relative importance of each of the three components described above. Flux-densities at wavelengths $\lambda<100 \mu \mathrm{m}$ define a slope much less steep than expected for a modified black-body curve. In particular flux-densities at $12 \mu \mathrm{m}$ and $25 \mu \mathrm{m}$ are much higher than expected for such a curve. Moreover, the steep rise in intensity from 12 to $25 \mu \mathrm{m}$ precludes any significant contribution by emission from PAH's, as the broadband spectrum of these decreases with wavelength. At the longwavelength side, the corrected flux-densities between 450 and $1200 \mu \mathrm{m}$ are nominally proportional to about $\lambda^{-2.5}$, i.e. proportional to a modified blackbody emission with a wavelength dependence $\beta \leq 1$, more characteristic for small than for large dust grains, or typical for a superposition of various large grain temperature components.

In order to further analyze the dust population of NGC 1569, represented by the observed emission, we have modelled the spectrum for a few representative cases. We present here two particular cases: (i) emission dominated by large particles at different temperatures and (ii) a three-component fit in which the relative fractions of different types of dust particles are varied.

\subsection{An abundance of cold dust?}

In principle, it is well possible to fit the emission spectrum over the entire observed range with large grains at various temperatures. As we have only seven observed points, we must limit ourselves to a model allowing for three different temperature components. Obviously, even better fits can be obtained by allowing for more temperature components, but there is no physical relevance to such an improvement. In Fig. 3 we show the result, adopting $\beta=2$. Table 2 presents the flux densities of the different components. The data can be fit very satisfactorily, which is no surprise given the large number of free parameters (almost equal to the number of independent data points). As Fig. 3 shows, each component is essentially determined by two different measured points. However, in order to reproduce the specific features at the short and the long wavelength 


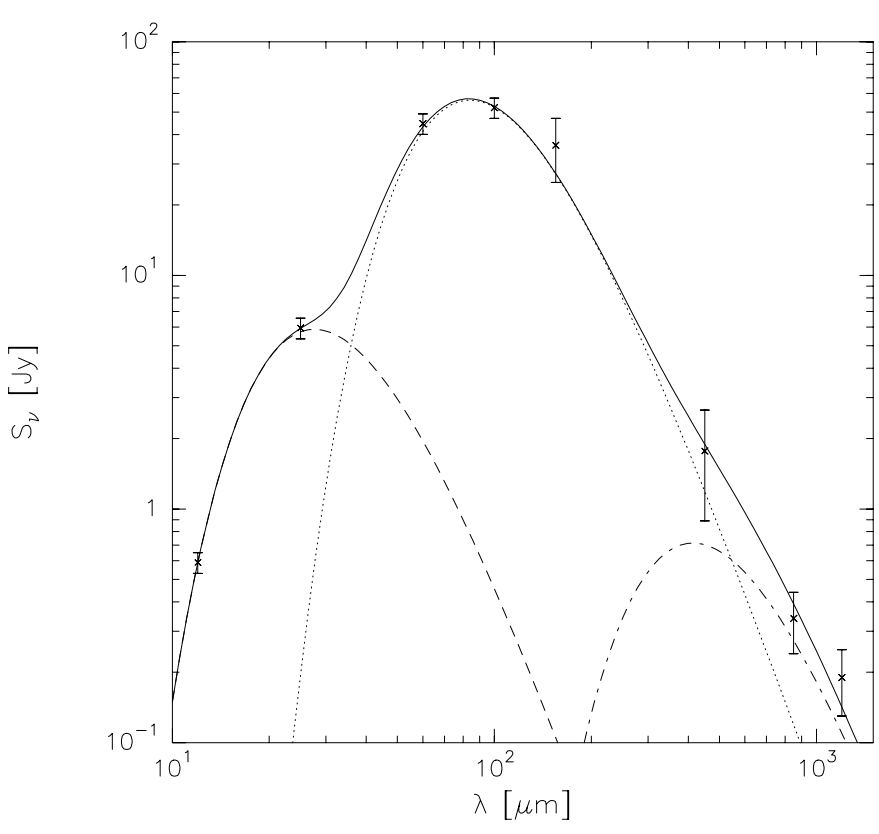

Fig. 3. The mid-infrared to millimetre spectrum of NGC 1569 , fitted with a three-temperature large-grain model. Crosses mark flux-densities from Table 1 . The three dust components have temperatures of $105 \mathrm{~K}$ (dashed line), $34.5 \mathrm{~K}$ (dotted line) and $7 \mathrm{~K}$ (dashed dotted line).

sides referred to in the previous section, the inclusion of both rather hot and rather cold dust components is unavoidable. The temperature of the coolest component required to fit the relatively high (sub)millimeter emission is very low, of the order of only $7 \mathrm{~K}$.

Moreover, because of the low dust emissivities at this temperature, a very large fraction of all dust would have to be so cold in order to explain the emission at $850 \mu \mathrm{m}$ and $1200 \mu \mathrm{m}$. From the large grain component fits to the NGC 1569 spectrum we may estimate the dust mass from each components $100 \mu \mathrm{m}$ emission:

$M_{\mathrm{d}}=\frac{D^{2} S_{100 \mu \mathrm{m}}}{B_{100 \mu \mathrm{m}}(T) k_{100 \mu \mathrm{m}}}$.

Here, $B_{100 \mu \mathrm{m}}(T)$ is the Planck function at $100 \mu \mathrm{m}$. We have assumed a dust extinction coefficient (per dust mass) of $k_{100 \mu \mathrm{m}}=63 \mathrm{~cm}^{2} \mathrm{~g}^{-1}$. Extrapolating this value with $k_{\lambda} \propto \lambda^{-2}$ and adopting a gas-to-dust mass ratio of 150 we obtain $k_{1200 \mu \mathrm{m}}=0.0025 \mathrm{~cm}^{2} \mathrm{~g}^{-1}$ (per gas mass) in good agreement with other studies (Krügel et al. 1990; Mezger et al. 1990; Draine \& Lee 1984). The results for the three components shown in Fig. 3 are a small mass of hot dust, $M_{\mathrm{d}, \mathrm{h}}=12.4 M_{\odot}$, a significant mass of warm dust, $M_{\mathrm{d}, \mathrm{w}}=3.1 \times 10^{4} M_{\odot}$ and a very large mass for the very cold dust: $M_{\mathrm{d}, \mathrm{c}}=1.14 \times 10^{6} M_{\odot}$. The total dust mass $M_{\mathrm{d}, \text { tot }}=1.17 \times 10^{6} M_{\odot}$ would thus be completely (i.e. for $97 \%$ ) dominated by the cold dust component.

How large is the uncertainty in this mass? The dust mass depends on the adopted dust temperatures and wavelength dependence of the extinction coefficient, $\beta$. The highest temperature of the cold dust that is just marginally consistent with the errors of the $450-1200 \mu \mathrm{m}$ data is $11 \mathrm{~K}$. This temperature is mainly determined by the slope of the spectrum between $450-1200 \mu \mathrm{m}$. With this, we derive a total dust mass of $5 \times 10^{5} M_{\odot}$, about a factor of 2 lower than derived for a dust temperature of $7 \mathrm{~K}$.

The actual dust mass depends strongly on the choice of $\beta$. For instance, if $\beta=1$, we could fit the dust emission longwards of $60 \mu \mathrm{m}$ with only one dust component at a temperature of about $45 \mathrm{~K}$ - no cold dust being required at all. The total dust mass would then be much lower, only about $10^{4} M_{\odot}$. However, we do not believe $\beta=1$ to be a plausible choice, because nearby galaxies provide compelling evidence (see Sect. 4.1) that $\beta \simeq 2$ for large grains. Thus, if we had $\beta \simeq 1$ in NGC 1569 , it would indicate that its dust properties are very different from other nearby galaxies, a possibility no different from the one discussed in Sect. 4.3.

In order to explore the influence of a slightly different $\beta$, which might still be consistent with the observations presented in Sect. 4.1, we have fitted the spectrum of NGC 1569 with $\beta=1.8$. The results are very similar to $\beta=2$. The hot dust component is unchanged and the warm and cold component require temperatures of 37 and $7 \mathrm{~K}$, respectively. The total dust mass is slightly reduced to $8.7 \times 10^{5} M_{\odot}$.

In conclusion, if $\beta \simeq 2$ as in all or most other nearby galaxies, then the dust mass necessary to explain the spectrum of NGC 1569 within a multi-temperature model is at least $5 \times 10^{5} M_{\odot}$ and, if we take the best-fit to the data, even higher, $1.2 \times 10^{6} M_{\odot}$.

\subsubsection{Where can the cold dust hide?}

Such a large amount of cold dust is a very unlikely state of affairs in a low-metallicity galaxy dominated by intense radiation fields. For instance, from the UV measurements in I88 we estimate a mean radiation field at $1000 \AA$ within NGC 1569 (assuming NGC 1569 to be spherical with a radius of 1 arcmin $=640 \mathrm{pc}$ ) of about $1 \times 10^{-16} \mathrm{erg} \mathrm{s} \mathrm{cm}^{-2} \mathrm{~Hz}^{-1}$. As the Solar Neighbourhood is characterised by a value of about $1 \times$ $10^{-18} \mathrm{erg} \mathrm{s}^{-1} \mathrm{~cm}^{-2} \mathrm{~Hz}^{-1}$ (Mezger et al. 1982), the NGC 1569 radiation field field is two orders of magnitude higher in good agreement with the strong thermal free-free and $\mathrm{H} \alpha$ emission from this post-starburst galaxy. Such high radiation field intensities suggest that dust temperatures should be a factor of $100^{1 / 5}=2.5$ (for $\beta=1$ ) to $100^{1 / 6}=2.1$ (for $\beta=2$ ) higher than for the Solar Neighbourhood. The latter has to be about $10 \mathrm{~K}$ for silicates and $18 \mathrm{~K}$ for graphite (Mathis et al. 1983) which would imply a minimum dust temperature of $20 \mathrm{~K}$ in NGC 1569. The same conclusion is drawn from a consideration of nearby spiral galaxies. Notwithstanding their higher metallicity (more shielding) and lower radiation field intensities, the coolest component contributing to their far-infrared/submillimetre spectra has 
a temperature of typically $15 \mathrm{~K}-20 \mathrm{~K}$ (e.g. NGC 891: Alton et al. 1998; Israel et al. 1999; M 51: Guélin et al. 1995, NGC 3627: Sievers et al. 1994). The coldest dust component in a galaxy such as NGC 1569 with very little shielding against very strong radiation fields should clearly be warmer than this.

We can estimate the global dust amount from an energy budget consideration by comparing the absorbed to the bolometric radiation. Integrating the UV flux densities from 1500-3650 $\AA$, the optical flux densities from 3650$5500 \AA$ and the infrared-to-millimetre flux densities from $12-1200 \mu \mathrm{m}$ we obtain $F_{\mathrm{UV}}=2.9 \times 10^{-9}, F_{\mathrm{opt}}=1.7 \times 10^{-9}$ and $F_{\text {dust }}=3.7 \times 10^{-9} \mathrm{erg} \mathrm{s}^{-1} \mathrm{~cm}^{-2}$.

We have to make a distinction between the dust emission from HII regions and the diffuse dust emission. The dust in HII regions is mainly heated by ionizing photons that are almost completely absorbed by the dust locally, practically independent of the dust amount. The diffuse dust, on the other hand, is mainly heated by nonionizing photons, and the amount of radiation absorbed is directly related to the dust opacity. Thus, in order to calculate the (diffuse) dust opacity, we have to subtract the dust heating by ionizing photon. We estimate the ionizing UV radiation from the $\mathrm{H} \alpha$ emission. Waller (1991) finds a total $\mathrm{H} \alpha$ luminosity of $L_{\mathrm{H} \alpha}=4.5 \times 10^{40} \mathrm{erg} \mathrm{s}^{-1}$. This value is corrected for the Galactic foreground extinction of $E(B-V)=0.56$ (I88); we neglect any extinction internal in the HII regions. The total dust emission originating from ionizing radiation can be estimated as $L_{\text {dust }}^{\text {ion }}=27.12 \times L_{\mathrm{H} \alpha}=$ $1.2 \times 10^{42} \mathrm{erg} \mathrm{s}^{-1}$ (Xu \& Buat 1995). The corresponding flux is $F_{\text {dust }}^{\text {ion }}=L_{\mathrm{H} \alpha} /\left(4 \pi D^{2}\right)=2.0 \times 10^{-9} \mathrm{erg} \mathrm{s}^{-1} \mathrm{~cm}^{-2}$.

The fraction of radiation absorbed by the diffuse dust is $\left(F_{\text {dust }}-F_{\text {dust }}^{\text {ion }}\right) /\left(F_{\mathrm{UV}}+F_{\text {opt }}+F_{\text {dust }}-F_{\text {dust }}^{\text {ion }}\right)=0.27$. We estimate the diffuse dust opacity using a simplified radiation transfer model with a slab geometry, assuming that dust and stars are homogeneously mixed. The dust mass derived in this geometry is higher than if the dust were in a foreground layer. We use an approximate formula $(\mathrm{Xu} \&$ de Zotti 1989) for the dust absorption probability, further simplified by applying for the UV and optical radiation the extinction properties at 2000 and $4300 \AA$, respectively (see Lisenfeld et al. 1996). With this we derive an opacity in the blue of $\tau_{B}=0.16$. Assuming that the dust has the same extinction properties as dust in our Galaxy (DBP90), we can estimate the dust mass (in $M_{\odot}$ ) as

$M_{\text {dust }}=8.8 \times 10^{4} \times \tau_{B} \times\left(\frac{A}{\mathrm{kpc}^{2}}\right)$,

where $\mathrm{A}$ is the surface area of the galaxy. Assuming a circular area with radius $80^{\prime \prime}$ we obtain $M_{\text {dust }}=2.3 \times$ $10^{4} M_{\odot}$. The uncertainty in this estimate is, even taking into account unknown parameters like the geometry of dust and stellar distribution, certainly less than a factor of 2 so that the dust mass derived is more than an order of magnitude lower than the cold dust necessary to explain the dust emission spectrum. If the dust contained more VSG's than in our Galaxy, the derived dust mass would be lower because the extinction efficiency per mass is higher for VSG's (DBP90).

The above considerations do not exclude the presence of very dense regions of cold dust barely contributing to the extinction or overall emission. But where could such a dust component be? It would have to be in regions of high dust opacity in order to be shielded from the interstellar radiation field (ISRF) or be situated far away from stars. The latter is not the case: NGC 1569 is a small galaxy with an intense star-formation over the whole area where dust emission is observed. In fact, the maximum of the dust emission is very close to the large star clusters A and B (Fig. 1). Is the self-shielding of the dust enough to maintain the inner parts cold? The opacity, $\tau$, along the line of sight can be calculated as:

$\tau_{\nu}=\frac{S_{\nu}}{\Omega B_{\nu}(T)}$

where $S_{\nu}$ is the flux density coming from the solid angle $\Omega$. For a dust temperature of $7 \mathrm{~K}(11 \mathrm{~K})$ we derive from the peak flux density of $25 \mathrm{mJy} /$ beam $\tau_{1200 \mu \mathrm{m}}=1.5 \times$ $10^{-3}\left(5.8 \times 10^{-4}\right)$. With $\tau_{V} / \tau_{1200 \mu \mathrm{m}}=4 \times 10^{4}$ (Kramer et al. 1998) this gives an optical opacity of $\tau_{V}=58$ (23). Maximum shielding could be achieved if this dust were in one big cloud illuminated from outside only. But also in this case, a rather thick outside layer of warm dust exposed to the ISRF will be present. In order to attenuate the ISRF of NGC 1569 by a factor of 100, which would make it comparable to the Galactic ISRF, a layer of $\tau_{V}=4.5$ is needed. Thus, we can estimate the cold dust mass fraction by considering a sphere with diameter $\tau_{V}=58$ (23) where an outer layer of $\tau_{V}=4.5$ is made of warm dust and the inner part consists of cold dust. The mass ratio of cold dust to total dust is $60 \%(T=7 \mathrm{~K})$, respectively $20 \%(T=11 \mathrm{~K})$. This is not enough to explain the dust emission spectrum for which $97 \%$ (respectively $94 \%$ for $T=11 \mathrm{~K}$ ) of the dust mass must be cold.

An alternative way to shield the dust could be molecular gas which is most likely abundant in the regions of maximum dust emission (see Sect. 5). However, molecular hydrogen is dissociated only by photons of energies above $11 \mathrm{eV}$, corresponding to radiation shortwards of about $1000 \AA$ so that the overall radiation field of NGC 1569 does not get significantly damped.

A final argument against a large amount of cold dust, is that if it were present, the resulting dust mass is of order one per cent of the gas mass in the same area, $M_{\text {gas }} \approx$ $9.2 \times 10^{7} M_{\odot}($ I88, SI01; see also Sect. 4.3.2). This metalpoor galaxy would thus have an usually high dust-to-gas ratio.

For these reasons, we reject any explanation of the farinfrared/submillimetre spectrum of NGC 1569 involving the presence of large amounts of cold dust.

\subsection{An abundance of very small grains?}

As an alternative, we have fitted the dust emission spectrum of NGC 1569 with the model by DBP90. In this 


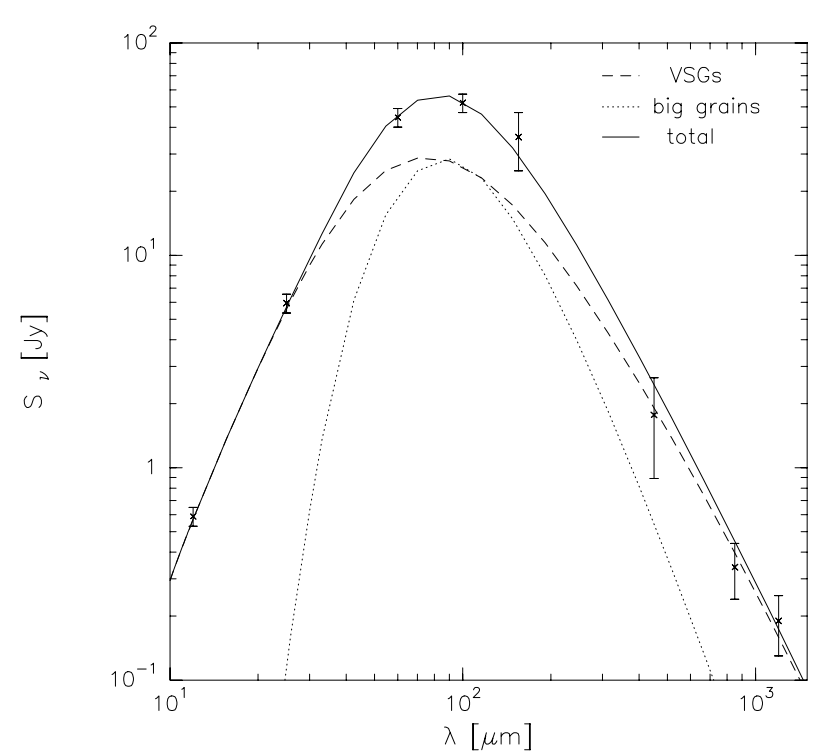

Fig. 4. The mid-infrared to millimetre spectrum of NGC 1569, fitted with the dust model of DBP90 assuming dust to be exposed to a radiation field equal to the Solar Neighbourhood field scaled up by a factor 60 . Emission by PAH's is negligible and the number of VSG's is increased by a factor of seven over the local values in DBP90. Crosses mark flux-densities from Table 1.

model, the dust is described by the three components mentioned above (PAH's, VSG's and large grains) exposed to a radiation field which can be varied in intensity. The maximum and minimum size, as well as the exponent of the size distribution of the various components can be varied. Here, we use those values for which DBP90 achieved the best-fit for the solar neighbourhood. The big grain size ranges from 15 to $110 \mathrm{~nm}$ and they are distributed in a power-law with exponent 2.9. The VSGs have sizes between 1.2 to $15 \mathrm{~nm}$ with a power-law exponent of 2.6. The corresponding values for PAHs are 0.4 to $1.2 \mathrm{~nm}$ and an exponent of 3 . The exponent of the wavelength dependence of the extinction coefficient is $\beta=2$ for large grains and $\beta=1$ for VSGs. We have tested different radiation fields and allowed for different relative contributions of the three components. We obtain a good fit for dust exposed to a radiation field similar in spectral shape to the local Solar Neighbourhood field, but with an intensity sixty times higher, consistent with the radiation field of NGC 1569 estimated in Sect. 4.2.

The resulting fit is shown in Fig. 4 and the flux densities of the various components are listed in Table 2. Not unexpectedly, the abundance of PAH's is found to be negligible because of the steep mid-infrared rise in the required spectrum. Madden (2000) reaches a similar conclusion from observations of the MIR/FIR spectrum of NGC 1569. However, the abundance of VSG's with respect to the large grains must be increased by a factors of about seven over the Galactic abundance assumed in the DBP90 model. The large VSG contribution is required to successfully match both the mid-infrared and the submillimetre/millimetre ends of the spectrum. The contribution of large grains is well-constrained by the requirement to fit the far-infrared data points.

The conclusion that the abundance of VSG's is enhanced with respect to the solar neighbourhood is very robust and does not depend on the details of the modelling, as we confirmed by trying to fit the data with other radiation fields given in DBP90 and changing the size distribution of the VSG's. Acceptable - although not equally good - fits could be achieved for the radiation field around an O5 star and for an enhanced UV radiation field. In those case the abundance by VSG's had to be increased by a factor of $2-3$.

Thus, there are two principal differences between our model fit of NGC 1569 and the model fits presented by DBP90 for the local Galactic environment. These are: (i) the almost total absence of PAH emission and (ii) a strong enhancement of the VSG contribution relative to that of the large grains by a factor of 2-\%. Both factors point to a different evolution of dust grains under the different environmental conditions prevalent in NGC 1569.

\subsubsection{Dust grain processing}

The first result is not entirely unique to NGC 1569. Both Magellanic Clouds also suffer from significant PAH depletion but not as strongly as NGC 1569 (Schwering 1988; Sauvage et al. 1990). However, it is relevant to note that, although the metallicity of NGC $1569(12+\log (\mathrm{O} / \mathrm{H})=$ 8.19, Kobulnicky \& Skillman 1997) is in between those of the LMC and the SMC, the intensity of its radiation field is 5-10 times higher. PAH depletion, in fact, appears to be a general characteristic of dwarf galaxies. In the compilation by Melisse \& Israel (1994), NGC 1569 clearly has the highest $f_{25} / f_{12}$ ratio (about 10 ), but it is followed by two more galaxies (NGC 3738 and IC 4662) with ratios of 7 and 8 respectively. The mean $f_{25} / f_{12}$ ratio of the remaining ten Im galaxies is $4.5 \pm 0.5$, whereas 15 larger galaxies classified $\mathrm{Sm}$ have a mean ratio $2.9 \pm 0.4$. Large late-type spiral galaxies with less intense mean radiation fields and higher metallicities mostly have ratios $1.5-2$. With the $f_{25} / f_{12}$ ratio as indicator, irregular dwarf galaxies clearly have dust contents significantly depleted in PAH's as compared to spiral galaxies. Most likely, PAH depletion is a function of the incident UV radiation, i.e. of the global UV radiation intensity and the amount of shielding available (Puget \& Léger 1989). In this respect, NGC 1569 provides one of the more extreme environments by being metal-poor and presently in a (post) starburst phase.

The magnitude of the increase in the VSG/large grain ratio with respect to that of the Solar Neighbourhood, suggests the amount of VSGs increase at the expense of large grains by extensive processing of the latter. Graingrain collisions in slow shocks can efficiently destroy large grains by fragmenting and shattering and convert them into small grains producing an excess of small particles as we believe to see in NGC 1569 (Borkowski \& Dwek 1995; Jones et al. 1996). NGC 1569 is a post-starburst galaxy. 
A large fraction of its interstellar medium is in chaotic motion with a rather high mean velocity dispersion of about $20 \mathrm{~km} \mathrm{~s}^{-1}$, and over a dozen radio supernova remnants can be identified in this small galaxy. Thus, shocks are necessarily present in its interstellar medium. In addition, the low metallicity of NGC 1569 may also hamper formation of large grains by inefficient accretion to begin with.

\subsubsection{Dust mass and gas-to-dust ratio}

The total dust mass derived from the adapted DBP90 model is much less than that found for the model based on cold large grains discussed in the preceding section. The mass of the large grains is derived from Eq. (1) with $S_{100 \mu \mathrm{m}}$ and $T$ being the flux density at $100 \mu \mathrm{m}$ of large grains and their temperature. The mass of the VSG component is found from the relative mass contributions of VSG's and large grains given by DBP90 (their Table 2), scaled up by the factor 7 that we have found for NGC 1569. Notwithstanding the substantial number increase of small grains with respect to large grains, the total dust mass $M_{\mathrm{d}, \mathrm{tot}}=3.2 \times 10^{4} M_{\odot}$ is still dominated by the large grains, with $M_{\mathrm{d}, \mathrm{LG}}=2.1 \times 10^{4} M_{\odot}(66 \%)$. The largegrain temperature required by the model fit $\left(T_{\mathrm{LG}}=32 \mathrm{~K}\right)$ is close to the temperature $\left(T_{\mathrm{IRAS}} \approx 35 \mathrm{~K}\right)$ that, ignoring everything else, we would obtain directly from the IRAS $f_{60} / f_{100}$ flux-density ratio. Partly because of this, the dust mass derived here is somewhat fortuitously not very different from the mass $2.8 \times 10^{4} M_{\odot}$ we would naively obtain from the IRAS $60 \mu \mathrm{m}$ and $100 \mu \mathrm{m}$ data only. This situation is very different indeed from the one pertinent to spiral galaxies where IRAS-derived dust masses commonly underestimate the total dust mass by an order of magnitude (Devereux \& Young 1990). The uncertainty in this mass derivation can be estimated by considering the masses derived for the full range of fits consistent with the errors. In no case a lower dust mass was derived. The highest dust mass derived (in a cases where a lower VSG abundance was needed) was $6.3 \times 10^{4} M_{\odot}$, i.e. not even a factor of 2 higher. The range in dust mass derived from the DBP90 model is thus $3.2-6.3 \times 10^{4} M_{\odot}$.

We have summed HI maps of NGC 1569 (SI01) over a circular area with radius of $80^{\prime \prime}$ comprising all the flux in the $1200 \mu \mathrm{m}$ map. We find an $\mathrm{HI}$ mass of $M_{\mathrm{HI}}=$ $5.1 \times 10^{7} M_{\odot}$. For mass contributions $M_{\mathrm{H} 2}=0.35 M_{\mathrm{HI}}$ (Israel 1997) and $M_{\mathrm{He}}=0.25 M_{\text {gas }}$, and a dust mass $M_{\mathrm{d}}=3.2-6.3 \times 10^{4} M_{\odot}$, we find an overall gas-to-dust ratio $M_{\text {gas }} / M_{\mathrm{d}} \approx 1500-2900$ which is significantly higher than the Solar Neighbourhood ratio. The corresponding ratio $M_{\mathrm{HI}} / M_{\mathrm{d}} \approx 1600$ is in good agreement with the prediction by Lisenfeld \& Ferrara (1998) that this ratio should exceed the Solar Neighbourhood value typically by an order of magnitude for metallicities $12+\log (\mathrm{O} / \mathrm{H})=8.70$ and 8.19 in the Milky Way and NGC 1569 respectively.

\section{Distribution of dust, atomic and molecular gas}

The (sub)millimetre maps not only closely resemble one another, but also maps of centimetre-wavelength radio continuum emission (Israel \& de Bruyn 1988; Wilding 1990) and $\mathrm{H} \alpha$ (Waller 1991) images. In all these maps emission peaks in the western part of the optical image of the galaxy, just west of "super" star cluster A (see Fig. 1). The $850 \mu \mathrm{m} / 1200 \mu \mathrm{m}$ continuum peak is close to the discrete CO clouds mapped by Greve et al. (1996) and Taylor et al. (1999), but offset from these by about $10^{\prime \prime}$ to the east. In all maps major emission curves eastwards, north of star clusters A and B, following a string of optically prominent HII regions (cf. Waller 1991). The overall extent of the (sub)millimetre continuum emission is very similar to the optical extent, and that at radio continuum wavelengths.

We might suspect that contamination of the (sub)millimetre continuum maps by free-free emission from ionized gas causes their resemblance to radio continuum and $\mathrm{H} \alpha$ maps. Indeed, in Sect. 3. we have shown that our maps at $1200 \mu \mathrm{m}$ and at $850 \mu \mathrm{m}$ contain non-negligible contributions of free-free emission. However, the fraction of global emission involved (25\% and $13 \%$ respectively) is insufficient to explain the resemblance especially at $850 \mu \mathrm{m}$. Nevertheless, the question thus arises whether the free-free emission is proportional to the dust emission over the entire maps. If this is not the case, specific structural details in our maps could indeed be due to local concentrations of free-free emission. In order to address these concerns, we have compared the $\mathrm{H} \alpha$ map by Waller (1991; his Fig. 3a) to our maps. The $\mathrm{H} \alpha$ contour values multiplied by factors of $5.1 \times 10^{-14}$ and $4.9 \times 10^{-14}$ should correspond to the free-free emission contribution across NGC 1569 in our $1200 \mu \mathrm{m}$ and $850 \mu \mathrm{m}$ maps. With these conversion factors, the $\mathrm{H} \alpha$ map shows that the free-free emission from ionized gas is on the whole reasonably well scaled with the thermal emission from dust. A notable exception is the main emission peak at $\alpha(2000)=4^{\mathrm{h}} 30^{\mathrm{m}} 47^{\mathrm{s}}, \delta(2000)=+64^{\circ} 50^{\prime} 58^{\prime \prime}$, coincident with the most prominent HII region complex of NGC 1569 (HII 2 in Fig. 1). The ionized gas should contribute about $12 \mathrm{mJy} /$ beam to the peak flux densities in our maps. This corresponds to disproportionate fractions of about $50 \%$ and $20 \%$ at $1200 \mu \mathrm{m}$ and $850 \mu \mathrm{m}$ respectively, about twice the global average. Below emission levels of $15-20 \mathrm{mJy} /$ beam $(1200 \mu \mathrm{m})$ and $40-50 \mathrm{mJy} /$ beam $(850 \mu \mathrm{m})$ ionized gas and dust emission are roughly proportional, equal to the global average.

We have convolved to the $16^{\prime \prime}$ JCMT $850 \mu \mathrm{m}$ resolution not only the IRAM $1200 \mu \mathrm{m}$ map, but also the WSRT HI column density map (SI01) (Fig. 5). The (sub)millimetre emission is mostly just south of the HI ridge in NGC 1569. The main peak, marking the location of molecular clouds and bright HII regions, is located on a saddle point in the HI distribution, i.e. close to a local minimum in the $\mathrm{HI}$ ridge, between the two elongated major HI maxima. We may use the maps for a direct comparison of the (sub)millimetre emission intensity with the column 


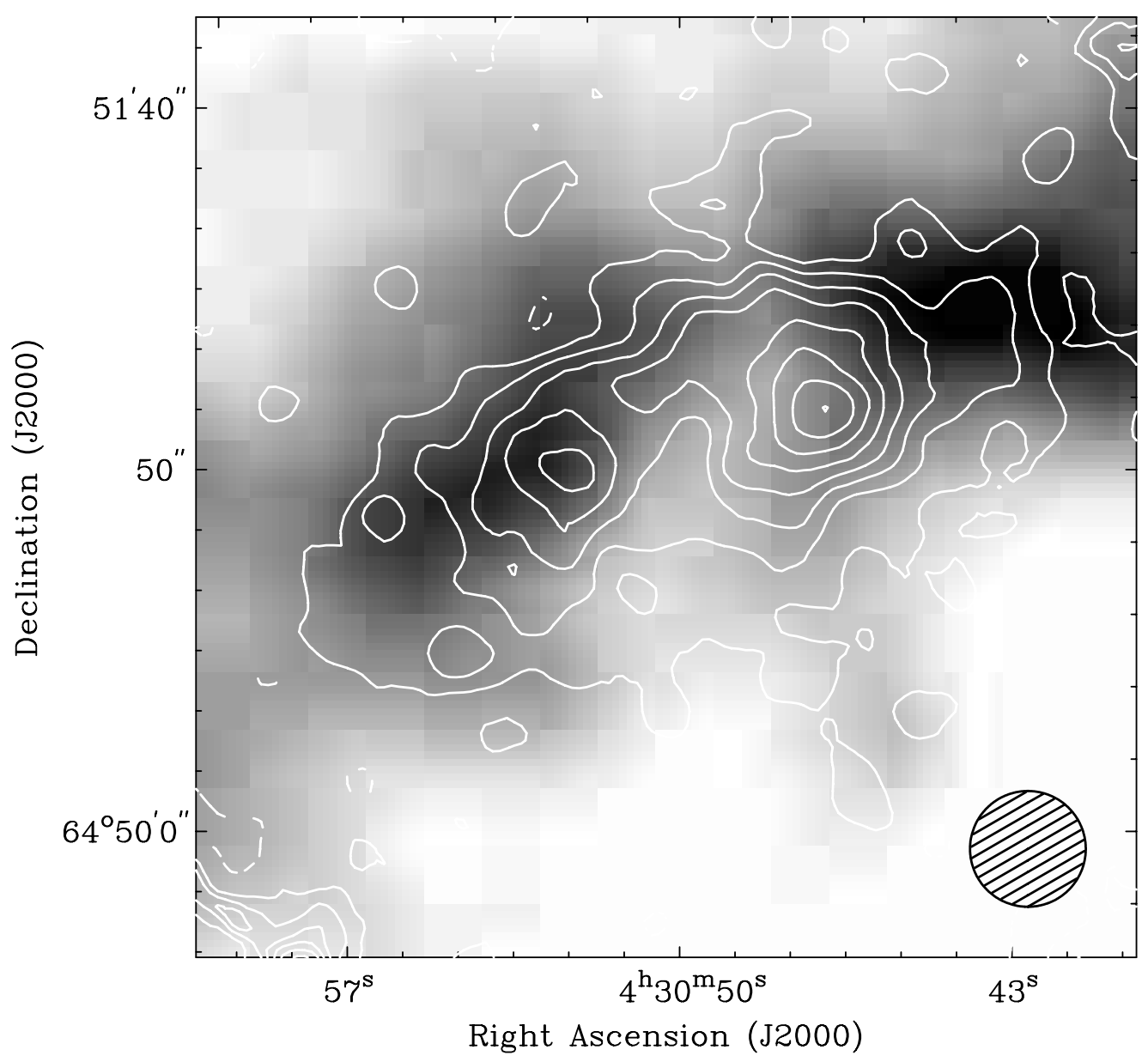

Fig. 5. Intensity at $850 \mu \mathrm{m}$ (contours) over HI column density (greyscales). Contour levels are $-8,8,16,24, \ldots$ mJy/beam. Grayscales increase linearly from $1 \times 10^{21}$ to $7 \times 10^{21} \mathrm{~cm}^{-2}$. The hatched circle indicates the size of the $16^{\prime \prime}$ beam $(F W H M)$ applicable to both maps.
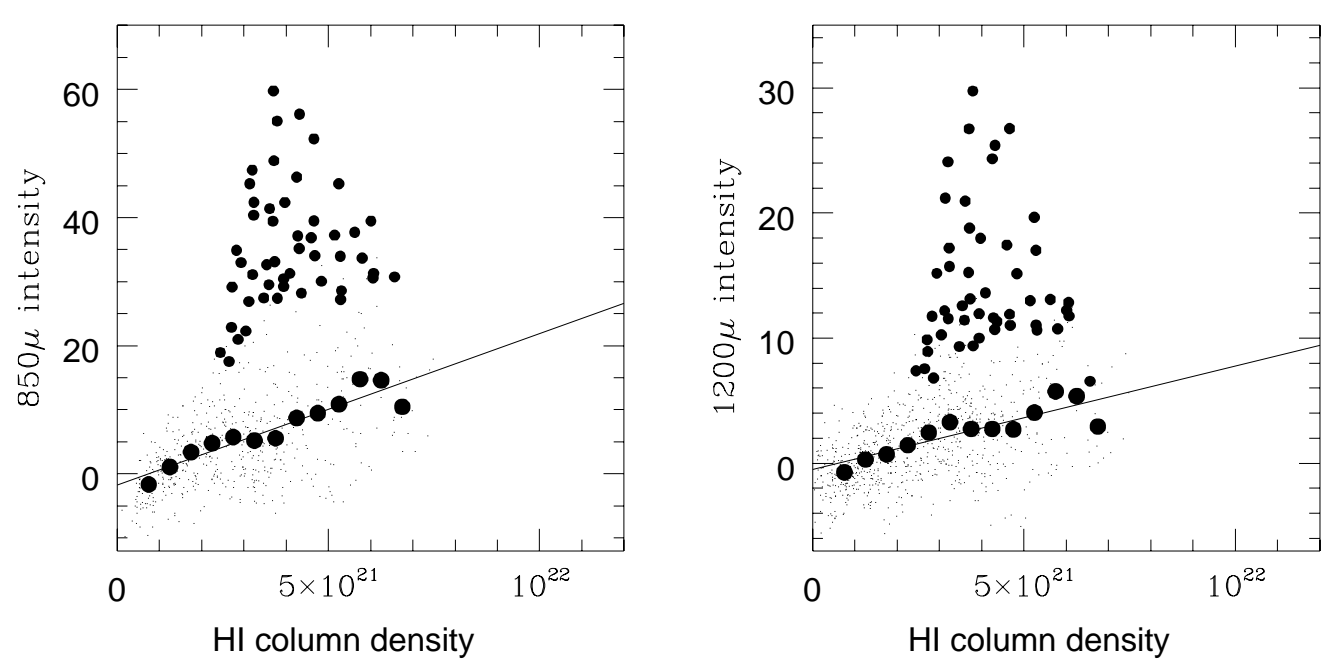

Fig. 6. Correlation between HI column density and intensity at $850 \mu \mathrm{m}$ (left) and $1200 \mu \mathrm{m}$ (right). Small and medium-sized black dots correspond to $5^{\prime \prime}$. The medium-sized dots mark positions where the 21 -cm radio continuum intensity is higher than $20 \mathrm{mJy} /$ beam. The large dots are binned averages of the remaining points (small dots) and the straight line is a fit to these average points. 
density of neutral hydrogen (HI). Figure 6 displays the correlation between the latter and the $850 \mu \mathrm{m}$ and $1200 \mu \mathrm{m}$ intensities. The overwhelming majority of points (small dots in Fig. 6) follows a linear relationship between broadband intensity and HI column density. At these intensity levels free-free emission is a more or less constant fraction of dust emission, and at these wavelengths most of the continuum emission is contributed by very small grains. Thus, the linear relation reflects a fairly constant VSG-to-gas ratio in NGC 1569. The relatively small fraction of points that deviate strongly from the general correlation is contributed entirely by the regions of most intense emission in the SCUBA/IRAM maps, notably the peak just mentioned.

In order to determine the general correlation, we first applied a cutoff for all points with more than $20 \mathrm{mJy} /$ beam in the $21-\mathrm{cm}$ radio continuum map; this effectively excludes all strongly deviating points. We then averaged the dust emission in $5 \times 10^{20} \mathrm{~cm}^{-2}$ intervals of $\mathrm{HI}$ column density. The resulting points are marked by large dots in Fig. 6. A fit to the points thus found, corrected for the free-free contribution, yields the relations $I_{1200}=$ $6.2 \times 10^{-22} N_{\mathrm{HI}}-0.51$ and $I_{850}=2.0 \times 10^{-21} N_{\mathrm{HI}}-1.71$, $\left(N_{\mathrm{HI}}\right.$ in $\mathrm{cm}^{-2}, I$ in mJy per 16 arcsec beam). Assuming a constant gas-to-dust mass ratio, and neglecting local temperature variations, the deviating points then seem to imply the presence of a large amount of undetected hydrogen, which should be in molecular form. This consideration allows us to estimate the molecular mass independently from uncertain CO-to- $\mathrm{H}_{2}$ conversion factors (Israel 1997).

The largest difference is found for the peak in the maps, with a mean intensity $46 \mathrm{mJy}$ per 16 arcsec beam at $850 \mu \mathrm{m}$ and $22 \mathrm{mJy}$ per beam at $1200 \mu \mathrm{m}$. Corrected for a mean free-free-contribution of $10 \mathrm{mJy} /$ beam (somewhat less than the free-free peak), these intensities imply total hydrogen column densities $N_{\mathrm{H}}=2 \times 10^{22} \mathrm{~cm}^{-2}$. The difference $N_{\mathrm{H}}-N_{\mathrm{HI}}$ is the column density of hydrogen atoms bound in molecules. Thus, Fig. 6 suggests a mean molecular hydrogen column density $N_{\mathrm{H}_{2}}=8 \times 10^{21} \mathrm{~cm}^{-2}$.

Division by the mean integrated $\mathrm{CO}$ intensity over this area, $I_{\mathrm{CO}} \approx 1.5 \mathrm{~K} \mathrm{~km} \mathrm{~s}^{-1}$ (Greve et al. 1996) yields an estimate for the actual CO-to- $\mathrm{H}_{2}$ conversion factor $X_{1569} \approx 5.3 \times 10^{21} \mathrm{~cm}^{-2}\left(\mathrm{~K} \mathrm{~km} \mathrm{~s}^{-1}\right)^{-1}$. This result, about 25-30 times the commonly adopted Galactic value, is very close to that obtained by Greve et al. (1996), but rejected by them; it is a factor of three below the high but uncertain value estimated by Israel (1997) and about a factor of three higher than the estimate by Taylor et al. (1999) which was derived from virial considerations and therefore should be considered a lower limit under the conditions prevalent in NGC 1569 (Israel 1997, 2000).

It is interesting to note that the deviating points in Fig. 6, implying the need of molecular gas, only occur for $N_{\mathrm{HI}} \geq 3 \times 10^{-21} \mathrm{~cm}^{-2}$. This could be due to a threshold in $N_{\mathrm{HI}}$ necessary for the formation of molecular gas. Such a threshold exists in our Galaxy around $N_{\mathrm{HI}} \approx 5 \times 10^{-20} \mathrm{~cm}^{-2}$ (Federman et al. 1979) and reflects the balance between molecular gas formation and destruc- tion by photodissociation. A minimum gas column density is necessary in order to protect the molecular gas from the radiation field through self-shielding as well as shielding by dust grains. It can be expected that in NGC 1569 the threshold $N_{\mathrm{HI}}$ is higher, in agreement with our finding, because the low dust content decreases both the shielding and the molecular gas formation rate and because the high radiation field increase the molecular gas destruction rate.

As can be seen in Fig. 5, adding these molecular hydrogen column densities to those of HI results in a total filling-in of the HI ridge minimum. It appears that the total hydrogen distribution in NGC 1569 remain ridge-like, but with a smooth increase to a pronounced maximum coincident with the present continuum peak.

\section{Conclusions}

1. We present new maps of the dwarf galaxy NGC 1569 at 450,850 and $1200 \mu \mathrm{m}$ taken with SCUBA at the JCMT and the MPIfR bolometer array at the IRAM $30 \mathrm{~m}$ telescope. Integrated flux-densities at these wavelengths may be compared to those at at 12, 25, 60 and $100 \mu \mathrm{m}$ obtained earlier with IRAS.

2. The steep rise in intensity from 12 to $25 \mu \mathrm{m}$ excludes a significant contribution from PAHs, as the broadband spectrum of these decreases in wavelength.

3. The (sub)millimetre flux densities are high compared to the flux densities at shorter wavelengths and the (sub)millimetre spectrum has a relatively shallow slope $\left(\simeq \lambda^{-2.5}\right)$. Such a spectral shape can be explained by the presence of a significant amount of cold dust. Fits to the observed spectrum by three-temperature dust models, however, require most dust to be at temperature of only about $7 \mathrm{~K}$.

4. We do not favour this explanation. The intense radiation field and low metallicity of NGC 1569, implying poor shielding, render it very unlikely that large amounts of dust at such low temperatures may exist in NGC 1569. We show that the high dust opacities necessary to shield a large fraction of the dust from this radiation field are not present. Even if it could, the resulting gas-to-dust mass ratio would have to be about 100 , again an unusually low value for a low metallicity galaxy such as NGC $1569(12+\log (\mathrm{O} / \mathrm{H})=8.19)$.

5. Alternatively, the (sub)millimetre flux densities may be dominated by emission of VSG's at various nonequilibrium temperatures. The combined emission is characterized by a wavelength dependence mimicking an extinction coefficient $k_{\lambda} \propto \lambda^{-1}$. Using the dust model of DBP90, we achieve good fits for dust exposed to radiation fields similar in spectral shape to the Solar Neighbourhood field but with sixty times higher intensity, as is appropriate for NGC 1569 (I88).

6. The fits require VSG to large grain abundances to be enhanced by a factor of 7 as compared to those in Solar Neighbourhood. The precise value of the enhancement factor is slightly model dependent; use of different 
radiation field yielding less good, but still acceptable fit results, yields slightly different factors. A robust conclusion is that the VSG enhancement factor in NGC 1569 is $2-7$.

7. Although in these models much of the emission originates from very small grains, virtually all of the mass still resides in the large grains. The gas-to-dust mass ratio is $1500-2900$, about an order of magnitude higher than in the solar neighbourhood.

8. Both the dust and molecular gas distributions peak at a local minimum in the $\mathrm{HI}$ ridge. If gas-to-dust mass ratio are constant over NGC 1569, the lack of $\mathrm{HI}$ at the very peak of the dust emission indicates the presence of a significant column of molecular gas. From the inferred molecular gas column density and the observed CO emission, we estimate the hydrogen column density to integrated $\mathrm{CO}$ intensity conversion factor $X \approx 5 \times 10^{21} \mathrm{H}_{2} \mathrm{~mol} \mathrm{~cm}{ }^{-2}\left(\mathrm{~K} \mathrm{~km} \mathrm{~s}^{-1}\right)^{-1}$, or about 25-30 times the local Galactic value.

Acknowledgements. We would like to thank F.-X. Désert for providing us with his program of the dust model, Martijn Kamerbeek for help in the data reduction and the JCMT staff, in particular Fred Baas, for carrying out the observations in service mode. We would like to thank the referee, E. Dwek, for many useful suggestions. This work made use of the NASA Extragalactic Database.

\section{References}

Ables, H. D. 1971, Publ. US. Naval Obs Sec. Ser., XX(IV), 61 Aloisi, A., Clampin, M., Diolaiti, E., et al. 2001, AJ, 121, 1425 Alton, P. B., Bianchi, S., Rand, R. J., et al. 1998, ApJ, 507, L125

Arp, H. C., \& Sandage, A. R. 1985, AJ, 90, 1163

Borokowski, K. J., \& Dwek, E. 1995, ApJ, 454, 254

Chini, R., Krügel, E., \& Steppe, H. 1992, A\&A, 255, 87

Désert, F.-X., Boulanger, F., \& Puget, J. L. 1990, A\&A, 237, 215 (DPB90)

De Vaucouleuers, G., De Vaucouleurs, A., Corwin, H. G., et al. 1991, Third Reference Catalogue of Bright Galaxies (Springer-Verlag, New York)

Devereux, N. A., \& Young, J. S. 1990, ApJ, 340, 708

Draine, B. T., \& Lee, H. M. 1984, ApJ, 285, 89

Dunne, L., \& Eales, S. A. 2002, MNRAS, in press [astro-ph/0106362]

Federman, S. R., Glassgold, A. E., \& Kwan, J. 1979, ApJ, 227, 466

Greve, A., Becker, R., Johansson, L. E. B., \& McKeith, C. D. 1996, A\&A, 312, 391

Greggio, L., Tosi, M., Clampin, M., et al. 1998, ApJ, 504, 725

Guélin, M., Zylka, R., Mezger, P. G., Haslam, C. G. T., \& Kreysa, E. 1995, A\&A, 298, L29

Holland, W. S., Robson, E. I., Gear, W. K., et al. 1999, MNRAS, 303, 659

Huchtmeier, W. K., Karachentsev, I. D., \& Karachentseva, V. E. 2000, in Small Galaxy Groups, IAU Coll. 174, ASP Conf. Ser., 209, 158

Hunter, D. A., Thronson, H. A., Casey, S., \& Harper, D. A. 1989, ApJ, 341, 697
Hunter, D. A., O'Connel, R. W., Gallagher, J. S., \& Smecker-Hane, T. A. 2000, AJ, 120, 2383

Israel, F. P. 1988, A\&A, 194, 24 (I88)

Israel, F. P., \& de Bruyn, A. G. 1988, A\&A, 198, 109

Israel, F. P., \& van Driel, W. 1990, A\&A, 236, 323

Israel, F. P. 1997, A\&A, 328, 471

Israel, F. P., van der Werf, P. P., \& Tilanus, R. P. J. 1999, A\&A, 344, L83

Israel, F. P. 2000, in Molecular Hydrogen in Space, ed. F. Combes, \& G. Pineau des Forêts (Cambridge Univ. Press.), 293

Jones, A. P., Tielens, A. G. G. M., \& Hollenbach, D. J. 1996, ApJ, 469, 740

Kramer, C., Alves, J., Lada, C., et al. 1998, A\&A, 329, L33

Krügel, E., Steppe, H., \& Chini, R. 1990, A\&A, 229, 17

Krügel, E., \& Siebenmorgen, R. 1994, A\&A, 228, 929

Krügel, E., Siebenmorgen, R., Zota, V., \& Chini, R. 1998, A\&A, 331, L9

Kobulnicky, H. A., \& Skillman, E. D. 1997, ApJ, 489, 636

Lisenfeld, U., Völk, H. J., \& Xu, C. 1996, A\&A, 306, 677

Lisenfeld, U., \& Ferrara, A. 1998, ApJ, 496, 145

Madden, S. C. 2000, in The Interplay between Massive stars and the ISM, ed. D. Schaerer, \& R. Delgado-Gonzalez, New Astron. Rev., 44, 249

Mathis, J. S., Rumpl, W., \& Nordsieck, K. H. 1977, ApJ, 217, 425

Mathis, J. S., Mezger, P. G., \& Panagia, N. 1983, A\&A, 128, 212

Meier, D. S., Turner, J. L., Crosthwaite, L. P., \& Beck, S. C. 2001, ApJ, 121, 740

Melisse, J. P. M., \& Israel, F. P. 1994, A\&A, 285, 51

Mezger, P. G., Mathis, J. S., \& Panagia, N. 1982, A\&A, 105, 372

Mezger, P. G., Wink, J. E., \& Zylka, R. 1990, A\&A, 228, 95

Mühle, S., Hüttemeister, S., Klein, U., \& Wilcots, E. M. 2001 in Dwarf Galaxies and their Environment, ed. K. S. de Boer, R.-J., Dettmar, \& U. Klein (Shaker Verlag), 161

Ossenkopf, V., \& Henning, T. 1994, A\&A, 291, 943

Prada, F., Greve, A., \& McKeith, C. D. 1994, A\&A, 288, 396

Puget, J. L., \& Leger, A. 1989, A\&AR, 27, 161

Sauvage, M., Thuan, T. X., \& Vigroux, L. 1990, A\&A, 237, 296

Schwering, P. B. W. 1988, Ph.D. Thesis, Leiden Univ. (NL)

Seki, J., \& Yamamoto, T. 1989, Ap\&SS, 72, 79

Siebenmorgen, R., \& Krügel, E. 1992, A\&A, 259, 614

Sievers, A. W., Reuter, H.-P., Haslam, C. G. T., Kreysa, E., \& Lemke, R. 1994, A\&A, 281, 681

Stil, J., \& Israel, F. P. 1998, A\&A, 337, 64

Stil, J., \& Israel, F. P. 2001, in preparation (SI01)

Taylor, C. L., Kobulnicky, H. A., \& Skillman, E. D. 1998, AJ, 116,2746

Taylor, C. L., Hüttemeister, S., Klein, U., \& Greve, A. 1999, A\&A, 349, 424

Tielens, A. G. G. M., \& Allamandola, L. J. 1987, in Interstellar Processes, ed. D. J. Thronson Jr. (Reidel, Dordrecht), 397

Vallenari, A., \& Bomans, D. J. 1996, A\&A, 313, 713

Waller, W. H. 1991, ApJ, 370, 144

Wilding, T. 1990, Ph.D. Thesis, University of Cambridge (UK)

Xu, C., \& de Zotti, G. 1989, A\&A, 225, 12

Xu, C., \& Buat, V. 1995, A\&A, 293, L65 